\title{
The role of cuboidal $\omega$ precipitates on $\alpha$ precipitation in a Ti-20V alloy
}

\author{
Yufeng Zheng ${ }^{1}$, Deep Choudhuri ${ }^{2}$, Talukder Alam ${ }^{2}$, Robert E.A. Williams ${ }^{1}$, Rajarshi \\ Banerjee $^{2}$ and Hamish L. Fraser ${ }^{1}$ *
}

${ }^{1}$ Center for the Accelerated Maturation of Materials and Department of Materials Science and Engineering, The Ohio State University, 1305 Kinnear Road, Columbus, OH 43212, USA.

${ }^{2}$ Department of Materials Science and Engineering, University of North Texas, Discovery Park Suite E-132, Denton, TX 76203

* Corresponding author: fraser.3@osu.edu, CEMAS, The Ohio State University, 1305 Kinnear Rd., Suite 100, Columbus, OH 43212-1177, USA

\begin{abstract}
The early stages of super-refined $\alpha$ phase precipitation, aided by the presence of precursory isothermal cuboidal $\omega$ precipitates, in a Ti-20V alloy, has been investigated by coupling conventional transmission electron microscopy (TEM), high resolution scanning transmission electron microscopy (HRSTEM), including energy dispersive spectroscopy (EDS), and atom probe tomography (APT). These complementary techniques, reveal for the first time, conclusive experimental evidence of nucleation of $\alpha$ precipitates at the $\omega / \beta$ interface and the attendant partitioning of elements.
\end{abstract}

Keywords: Titanium alloy; Phase transformation; $\omega$ phase; High resolution scanning transmission electron microscopy; Atom probe tomography

$\omega$ precipitates in $\beta$-rich $\mathrm{Ti}$ alloys are typically associated with embrittlement and poor mechanical properties of the alloy [1-3]. Nevertheless, the presence of precursory metastable $\omega$ precipitates promotes a homogeneous intra-granular distribution of finescale $\alpha$ precipitates within the parent $\beta$ matrix $[4,5]$, and the resultant microstructure exhibits a good combination of strength and ductility [6,7]. Thus, $\omega$-assisted $\alpha$ precipitation holds tremendous potential to improve the mechanical properties of $\beta$ rich titanium alloys and, consequently, $\omega$-assisted $\alpha$ nucleation has been the subject of multiple previous investigations [4-6, 8-16].

The morphology of $\omega$ precipitates is dependent on the $\beta-\omega$ misfit. Low misfit alloys typically exhibit ellipsoidal $\omega$ precipitates while for higher misfits, the morphology is typically cuboidal. Various groups of researchers have proposed different theories to explain the influence of ellipsoidal $\omega$ precipitates on subsequent $\alpha$ precipitation in low misfit alloys $[6,8,9]$. More recently, two novel $\omega$-assisted $\alpha$ precipitation mechanisms have been introduced $[4,5,10,11]$. T. Li et al claim that oxygen enriched region at isothermal $\omega / \beta$ interface acts as the potent nucleation site of $\alpha$ and $\omega$ transform to $\alpha$ by a mixed displacive-diffusional mode based on some rather questionable experimental results. The HAADF-STEM image shown in Fig. 6 in reference [11] only shows the atomic configuration within a relatively small local area, and cannot clearly indicate the presence and contact of $\omega$ and $\alpha$ phases, without the corresponding fourier transform. Judging from periodic atom column intensity change in the figure, the claimed $\alpha$ phase region could be actually $\omega$ phase. And also the morphology of either $\omega$ phase or $\alpha$ phase shown in Fig. 10 in reference [11] are not consistent with the dark 
field image shown in this paper, and what is claimed as 'needle/plate' morphology of isothermal $\omega$ precipitates is rather unusual and contrary to the widely recognized ellipsoidal morphology of isothermal $\omega$ phase in low misfit titanium alloys. Therefore, the oxygen and aluminum concentration measurements associated with these claimed $\omega$ precipitates, and the proposed mechanism of oxygen segregation induced $\alpha$ precipitation, are not conclusive. On the other hand, in our recent study in Ti-5Al$5 \mathrm{Mo}-5 \mathrm{~V}-3 \mathrm{Cr}$ (Ti-5553) alloy, the influence of $\omega$ phase on the nucleation and growth of $\alpha$ precipitate has been systematically studied coupling advanced electron microscopy and phase field modeling. Three different size scales of $\alpha$ precipitates, namely refined, more-refined and super-refined, resulting from non-conventional transformation pathways, have been identified and characterized in detail in Ti-5553 $[4,5,17]$. Refined $\alpha$ precipitates are formed in the absence of any effect of precursor athermal $\omega$ precipitates, but due to thermally induced compositional fluctuations within the $\beta$ matrix via the pseudo-spinodal decomposition mechanism [17-20]. Morerefined and super-refined $\alpha$ preciptates are formed depending on indirect [5] and direct [4] influence, respectively, of precursor uniformly distributed metastable $\omega$ precipitates in the $\beta$ matrix. The prior nano-scale isothermal $\omega$ precipitates can alter the local structure and composition and consequently provide an additional driving force for subsequent $\alpha$ precipitation, and both these mechanisms have been validated by coupling high resolution STEM imaging, atom probe tomography, and phase field simulations [4].

In contrast, in systems exhibiting a high mismatch between the $\omega$ and $\beta$ phases, it has been proposed that $\alpha$ precipitates nucleate on ledges and misfit dislocations at the $\omega / \beta$ interface [12-16]. Examples of such systems include the binary Ti-V alloys $[12,15$, 16] as well as the commercial Ti-10V-2Fe-3Al alloy [16]. However, there is no conclusive experimental evidence presented in the published literature to validate this proposed mechanism. In most previous reports, the contact between $\omega$ and $\alpha$ precipitates has typically been shown in dark-field images, which are twodimensional projections, and may not be fully representative. While phase contrast high-resolution TEM images of $\omega / \alpha / \beta$ regions in mutual contact were shown in previous papers $[8,10,16]$, it is rather difficult to clearly discern the atomic scale structure in these images, and therefore one cannot conclusively establish the mutual contact between these phases. It is also interesting to point out that if indeed the $\omega$ assisted $\alpha$ precipitation mechanism proposed by $\mathrm{T}$. Li et al in $[10,11]$ is correct, such a mechanism should be also be applicable to high $\beta-\omega$ mismatch titanium alloys, because the stress field near the $\omega / \beta$ interface is even higher in such systems and could potentially strongly restrict the oxygen near $\omega / \beta$ interface as suggested by $\mathrm{T}$. Li et al in [10].

Therefore, the present study focuses on investigating the nucleation and growth of $\alpha$ precipitates in the presence of precursor well-developed cuboidal $\omega$ precipitates in a model Ti-20V (wt\%) alloy, using high fidelity characterization: $\mathrm{C}_{\mathrm{s}}$ (aberration)corrected atomic resolution Titan S/TEM, Super-XEDS and atom probe tomography (APT). The major objective of this investigation is to unambiguously provide direct experimental evidence showing the nucleation site of $\alpha$ precipitates in this Ti-20V alloy and to also explore the possible correlation between oxygen segregation and $\alpha$ precipitation. 
The Ti-20V alloy was prepared via arc-melting and was then homogenized at $1000^{\circ} \mathrm{C}$ for 7 days in a vacuum furnace. $5 \mathrm{~mm} \times 5 \mathrm{~mm} \times 5 \mathrm{~mm}$ cube samples were cut from the center of bulk material for subsequent heat treatment. The cut samples were $\beta$ solutionized at $900^{\circ} \mathrm{C}$ for 1 hour followed by water quench to room temperature. Subsequently $\beta$-solutionized samples were directly isothermally annealed at $375^{\circ} \mathrm{C}$ for 15 hours and 50 hours and after each annealing time the samples were water quenched to room temperature in order to conserve the microstructure formed. TEM foils were prepared from the center of heat treated samples using Focused Ion Beam (FIB), more specifically the FEI Nova 200 NanoLab and were cleaned at low voltage using Fischione Model 1040 Nanomill. TEM diffraction patterns and dark field images were completed on FEI/Philips CM200 TEM operating at 200kV. HAADFHRSTEM images were taken using probe-corrected FEI Titan ${ }^{3 T M} 80-300$ at $300 \mathrm{kV}$. Scanning transmission electron microscopy- X-ray energy dispersive spectroscopy (STEM-XEDS) mapping was collected in image-corrected $\operatorname{Titan}^{3 \mathrm{TM}}$ G2 60-300

(S)TEM equipped with FEI's ChemiSTEM technology. High angle, annular dark-field (HAADF) imaging was performed on the latter instrument using a convergence semiangle of $12 \mathrm{mrad}$ at a camera length of $91 \mathrm{~mm}$ with an incident beam dwell time of 4 $\mu \mathrm{s} / \mathrm{px}$ and a current of $\sim 90 \mathrm{pA}$; inner and outer detection angles were $34.8 \mathrm{mrad}$ and $230.0 \mathrm{mrad}$, respectively. 3D atom probe tomography was carried out in a LEAP $3000 \mathrm{x}$ HR, using voltage mode at a temperature of $60 \mathrm{~K}$, with an evaporation rate $0.2-1.0 \%$ and a voltage pulse fraction at $20 \%$ of steady-state applied voltage.

The results presented here primarily focus on two heat-treatments: as-quenched Ti$20 \mathrm{~V}$ annealed at $375^{\circ} \mathrm{C}$ for 15 hours to form well-developed isothermal cuboidal $\omega$ precipitates, and longer term annealed $\left(375^{\circ} \mathrm{C} / 50\right.$ hours $)$ to form $\alpha$ precipitates. A $[110]_{\beta}$ selected area diffraction pattern (SADP), and the corresponding dark field image from the Ti-20V sample after isothermal annealing at $375^{\circ} \mathrm{C}$ for 15 hours are shown in Figs. 1(a) and 1(b) respectively. Clear $\omega$ reflections were noted at the $1 / 3$ and $2 / 3\{112\}_{\beta}$ in the $[110]_{\beta}$ zone axis SADP, highlighted by red circles in Fig. 1(a). The corresponding dark field image in Fig. 1(b), recorded by selecting the set of four $\omega$ reflections, shows a homogeneous distribution of coarse ( $40 \mathrm{~nm}$ diameter) $\omega$ precipitates exhibiting a well-developed cuboidal morphology within the $\beta$ matrix. After annealing at $375^{\circ} \mathrm{C}$ for 50 hours, the [110] $]_{\beta}$ SADP and dark field image are shown in Figs. 1(c) and 1(d) respectively. In the $[110]_{\beta}$ SADP, strong $\omega$ reflections are observed clearly with the presence of very weak reflections close to $1 / 2\{112\}_{\beta}$ locations (indicated with arrows in Fig. 1(c)) that can be attributed to the early stages of $\alpha$ precipitation. A dark-field image recorded by including both $\omega$ and $\alpha$ reflections (highlighted with dashed circle in Fig. 1(c)), is shown in Fig. 1(d). After 50 hours annealing at $375^{\circ} \mathrm{C}$, the $\beta$ matrix consisted of coarse $\omega$ precipitates, and very fine $\alpha$ precipitates exhibiting a "diamond-like" morphology. Note that the low number density and volume fraction of super-refined $\alpha$ precipitates are consistent with the low intensity of $\alpha$ reflections in the $[110]_{\beta}$ SADP shown in Fig. 1(c). However, compared to the previously reported $\omega$-assisted super-refined $\alpha$ microstructure in Ti-5553[4], the number density of $\alpha$ precipitates in Ti-20V at initial nucleation stage is much lower, which can be related to the coarser size scale of isothermal $\omega$ particles in case of the current alloy. The dark-field image in Fig. 1(d) further shows the location of $\alpha$ 
precipitates (indicated with arrows) with respect to $\omega$ particles. In one case, a $\alpha$ precipitate appears to bridge two physically distinct $\omega$ particles (dashed circle), while in the other case a $\alpha$ particle seems to protrude out from a $\omega$ particle (dashed rectangle). It may be pointed out that the dark field image parallel to $[110]_{\beta}$ in Fig. $1(d)$ only suggests that $\alpha$ formation is linked with the pre-existing $\omega$ precipitates, Based on this evidence it is rather difficult to conclude that $\alpha$ and $\omega$ precipitates are in physical contact with each other (previously claimed as unambiguous evidence in [10, 11]), because a dark-field TEM image is essentially a $2 \mathrm{D}$ projection of a $3 \mathrm{D}$ microstructure, and, additionally, is inherently limited in spatial resolution.

Therefore, to reveal the 3D morphology at these length scales, APT investigations were coupled with the TEM studies. Fig. 2(a-d) show the results from a reconstructed atom probe tip, extracted from a Ti-20V specimen subjected to $375^{\circ} \mathrm{C} / 50$ hours annealing. The ion map in Fig. 2(a) shows titanium (Ti, in blue) and vanadium (V, in red) ions, and indicates that the $\alpha$ and $\omega$ phases (marked in Fig. 2(a)) are Ti-rich (V lean), while the surrounding $\beta$ regions are V-rich (or Ti-lean). The morphologies and size scales of the $\alpha$ and $\omega$ precipitates in the APT reconstruction, shown in Fig. 2(b) have been delineated by selecting a $84 \%$ Ti iso-surface, and correlate well with the dark-field image shown in Fig. 1(d). Thus, the APT ion map confirms that the $\alpha$ precipitates are in very close proximity with the $\omega$ precipitates in three dimensions. 1D composition profiles for vanadium and oxygen, across $\alpha / \beta$ and $\omega / \beta$ interfaces, averaged over a cylinder of diameter $3 \mathrm{~nm}$, are shown in Figs. 2(c-d). The actual locations of these cylinders, within the APT reconstructions, are also shown as insets. The statistical error bars in these composition profiles have also been indicated. As evident from the ion map (Fig. 2(a)), the composition profile shows that $\alpha$ and $\omega$ have much lower $\mathrm{V}$ content $(\sim 5$ at $\% \mathrm{~V})$ than the surrounding $\beta$ regions (as high as $\sim 35$ at $\% \mathrm{~V})$. To corroborate the APT observations, STEM-XEDS was performed. Fig. 2(e) shows a HAADF image of a region with a $\alpha$ precipitate adjacent to two $\omega$ particles, and Fig. 2(f) is the V map in the same area revealed in Fig. 2(e). Consistent with APT results, the STEM-XEDS mapping qualitatively indicated that both $\alpha$ and $\omega$ are Vlean with nominally similar compositions, while the $\beta$ matrix is substantially richer in V. Quantitatively, the 1D composition profiles for V in Figs. 2(c-d) demonstrate that both $\omega$ and $\alpha$ have similar nominal $\mathrm{V}$ content, indicating that the $\alpha$ precipitates may nucleate with the composition close to that of $\omega$ precipitates. Furthermore, we also note that the oxygen content in $\alpha, \omega$, and $\beta$ phases (see oxygen profile in Fig. 2(c-d)) do not exhibit any significant variation (all data points within the statistical error bars), suggesting that there is no significant partitioning of oxygen between these phases at this stage of the phase transformation. Therefore, the role of oxygen partitioning in the $\omega$-assisted nucleation and growth of $\alpha$ precipitates, as claimed in $[10,11]$, needs to be investigated in further detail.

However, the APT results do not contain structural or crystallographic information at the true atomic length scale. Therefore, the structure near the interface between a well-developed cuboidal $\omega$ precipitate and the adjacent $\beta$ matrix was investigated using atomic resolution aberration-corrected HAADF-STEM. As shown in Fig. 3, distinct ledges and terraces are clearly visible at the $\omega / \beta$ interface, recorded from the Ti-20V sample annealed at $375^{\circ} \mathrm{C}$ for 15 hours, with the incident beam parallel to $[110]_{\beta} / /[11 \overline{2} 0]_{\omega}$, and these have been marked along the interface. Terraces are 
composed of non-uniformly spacing $(1 \overline{1} 2)_{\beta}$ atom planes and ledges exhibit the same height connecting different terraces. Aberration-corrected HAADF-STEM images from the sample annealed at $375^{\circ} \mathrm{C}$ for 50 hours, viewed along the $[110]_{\beta}$ direction are shown in Fig. 4. Figs. 4(a) and (b) show lower and higher magnification views respectively, of co-existing $\beta, \omega$, and $\alpha$ phases in this sample. Consistent with the TEM image shown in Fig. 1(d), the lengths of $\alpha / \omega$ and $\beta / \alpha$ interfaces are comparable, resulting in the morphology of $\alpha$ precipitates to vary from the conventional plate-like morphology to more of a "diamond-like" morphology, partially constrained by the $\beta$ channel within which the $\alpha$ precipitate grows. Along the $\omega / \beta$ interface, a very thin layer of HCP structure $\alpha$ phase is highlighted by red color dashed line. The above result reveals that $\alpha$ precipitate does not nucleate in $\beta$ matrix and grows to contact $\omega$ particle, because if so the formed $\alpha$ plate should exhibit plate morphology in which the length of broad face is much longer than that of edge face. Therefore, Fig. 4(a) indicates clearly that the $\omega / \beta$ interfaces act as potential $\alpha$ nucleation sites, and interestingly, it appears that the $\alpha$ phase, once nucleated, grows into the $\beta$ phase. The notion of the growth of $\alpha$ plates into the $\beta$ phase is not in concert with some previous reports (e.g., Ohmori, et al. [16]), where it has been proposed that the $\alpha$ phase is nucleated and grows into the $\omega$ phase. However, it is consistent with previous work on nucleation of the $\alpha$ phase in the alloy Ti-5553, where it has been shown unambiguously that incipient plates of $\alpha$, while nucleated at $\beta / \omega$ interfaces grow into the $\beta$ phase prior to thickening ([4], Fig. 6). Presumably, as a given $\alpha$ plate increases in size, its thickening will consume the $\omega$ particles whose interfaces were involved in its nucleation event. The $\alpha / \omega / \beta$ interfaces have been marked in both figures, and the rectangular region (dotted line in Fig. 4(a)) marks the $\alpha / \omega / \beta$ triple junction that is shown at higher magnification in Fig. 4(b) at atomic resolution. Comparison of crystallographic axes of various phases unequivocally establishes the following expected $\alpha / \omega$ and $\alpha / \beta$ orientation relationships:

$$
\begin{gathered}
(11 \overline{2} 0)_{\omega} / /(0001)_{\alpha} \text { and }[0001]_{\omega} / /[\overline{1} 2 \overline{1} 0]_{\alpha} \\
(0001)_{\alpha} / /(110)_{\beta} \text { and } / /[\overline{1} 2 \overline{1} 0]_{\alpha} / /[\overline{1} 11]_{\beta}
\end{gathered}
$$

Furthermore, a careful examination reveals that while the $\alpha / \omega$ interface is composed of terrace and ledges, similar to comparatively jagged $\alpha / \beta$ interfaces highlighted, which could explain the comparable growth rate of $\alpha$ precipitate along these two interfaces. These HAADF-STEM results at atomic resolution clearly demonstrate that a direct physical contact exists between the $\alpha$ and $\omega$ phases with a specific orientation relationship; presenting direct experimental evidence that $\alpha$ precipitates can nucleate and grow heterogeneously from the $\omega / \beta$ interface.

The experimental results presented in this paper lead to novel insights into the nucleation mechanism of super-refined $\alpha$ precipitates from pre-existing cuboidal $\omega$ precipitates in a model Ti-20V alloy. The growth and coarsening of the cuboidal $\omega$ precipitates result in jagged (with terraces and ledges) $\omega / \beta$ interfaces. While these interface ledges are responsible for the growth of the $\omega$ precipitates, the same ledges can also act as potential sites for the nucleation of $\alpha$ precipitates. After longer term annealing, the $\alpha$ precipitates heterogeneously nucleate at the $\omega / \beta$ interfaces with both $\alpha$ and $\omega$ phases having similar solute content due to the rejection of vanadium into adjacent $\beta$ matrix. These $\alpha$ precipitates form a nearly coherent interface with both the $\omega$ precipitate as well as the $\beta$ matrix, and grow along the $\alpha / \omega$ and $\alpha / \beta$ interfaces at 
similar rates to produce a "diamond-like" morphology. The atom probe results presented in this paper, clearly reveal direct contact between the $\alpha$ and $\omega$ precipitates in three dimensions, and also show similar oxygen content in both $\alpha$ and $\omega$ phases without any apparent pile-up at or near the interface, indicating the minimal role played by oxygen on $\alpha$ nucleation. Thus, the mechanism of $\omega$-assisted $\alpha$ nucleation in Ti-V alloys, with a high $\beta-\omega$ misfit, could be dominated by defects such as ledges at the $\omega / \beta$ interface that act as favorable heterogeneous nucleation sites for super-refined $\alpha$ precipitates. More detailed investigations are presently underway, addressing the influence of $\omega / \beta$ interfaces on $\alpha$ precipitation by coupling phase field simulations and density-functional theory (DFT) based first principles calculations which will be discussed in future papers.

\section{Acknowledgements}

This research has been supported by the National Science Foundation, Division of Materials Research, grant \# DMR-1309270.

\section{References}

[1] A.W. Bowen, Scripta Metal., 5 (1971) 709-715.

[2] M.K. Koul, J.F. Breedis, Metal. Trans., 1 (1970) 1451-1452.

[3] J.C. Williams, B.S. Hickman, H.L. Marcus, Metal. Trans., 2 (1971) 1913-1919.

[4] Y. Zheng, R.E.A. Williams, D. Wang, R. Shi, S. Nag, P. Kami, J.M. Sosa, R. Banerjee, Y. Wang, H.L. Fraser, Acta Mater., 103 (2016) 850-858.

[5] Y. Zheng, R.E.A. Williams, J.M. Sosa, T. Alam, Y. Wang, R. Banerjee, H.L. Fraser, Acta Mater., 103 (2016) 165-173.

[6] O.M. Ivasishin, P.E. Markovsky, S.L. Semiatin, C.H. Ward, Mat. Sci. Eng. A., 405 (2005) 296-305.

[7] S.A. Mantri, D. Choudhuri, A. Behra, J.D. Coton, N. Kumar, R. Banerjee, Matel. Mater. Trans. A, 46 (2015) 2803-2808.

[8] F. Prima, P. Vermaut, G. Texier, D. Ansel, T. Gloriant, Scripta Mater., 54 (2006) 645-648.

[9] S. Nag, R. Banerjee, R. Srinivasan, J.Y. Hwang, M. Harper, H.L. Fraser, Acta Mater., 57 (2009) 2136-2147.

[10] T. Li, J.M. Cairney, D. Kent, M.S. Dargusch, D. Kent, G. Sha, Scripta Mater., 104 (2015) 75-78.

[11] T. Li, D. Kent, G. Sha, L.T. Stephenson, A.V. Ceguerra, S.P. Ringer, M.S. Dargusch, J.M. Cairney, Acta Mater., 106 (2016) 353-366.

[12] M.J. Blackbur, J.C. Williams, Tran. Met. Soc. AIME, 242 (1968) 2461-2469.

[13] C.G. Rhodes, J.C. Williams, Metal. Trans. A, 6 (1975) 2103-2114.

[14] J.C. Williams, M.J. Blackbur, Transactions of the Metallurgical Society of Aime, 245 (1969) 2352-2355.

[15] G.M. Pennock, H.M. Flower, D.R.F. West, Titanium' 80: Science and Technology, (1980) 1343-1351.

[16] Y. Ohmori, T. Ogo, K. Nakai, S. Kobayashi, Mater. Sci. Eng. A, 312 (2001) 182188.

[17] Y. Zheng, J.S. Sosa, R.E.A. Williams, Y. Wang, R. Banerjee, H.L. Fraser, Scripta Mater., 111 (2016) 81-84.

[18] S. Nag, Y. Zheng, R.E.A. Williams, A. Devaraj, A. Boyne, Y. Wang, P.C. Collins, G.B. Viswanathan, J.S. Tiley, B.C. Muddle, R. Banerjee, H.L. Fraser, Acta Mater., 60 (2012) 6247-6256. 
[19] A. Boyne, D. Wang, R.P. Shi, Y. Zheng, A. Behera, S. Nag, J.S. Tiley, H.L. Fraser, R. Banerjee, Y. Wang, Acta Mater., 64 (2014) 188-197.

[20] D. Wang, R. Shi, Y. Zheng, R. Banerjee, H.L. Fraser, Y. Wang, JOM, 66 (2014) 1287-1298.

\section{Figures Captions}

Figure 1. TEM results showing $[110]_{\beta}$ zone axis diffraction pattern, and the corresponding dark field images from specimens annealed at $375^{\circ} \mathrm{C}$ for 15 hours (a-b) and $375^{\circ} \mathrm{C}$ for 50 hours (c-d).

Figure 2. Compositional characterization after annealing at $375^{\circ} \mathrm{C}$ for 50 hours using APT (a-d) and STEM-XEDS (e-f). APT results show (a) reconstructed atom probe tip via blue Ti and red-V ions; (b) red-V ions and 84\% Ti iso-surface; $1 \mathrm{D}$ composition profile across (c) $\alpha / \beta$ interface and (d) $\omega / \beta$ interface. STEM-XEDS results show (e) HAADF image showing the contact of $\alpha$ precipitate and $\omega$ particles, and (f) V map showing vanadium is rejecting from both $\omega$ and $\alpha$ phase into $\beta$ matrix.

Figure 3. HAADF-STEM image showing ledges and terraces at the $\omega / \beta$ interface.

Figure 4. (a) Low magnification and (b) high magnification HAADF-STEM images, showing the nucleation site of $\alpha$ precipitates 


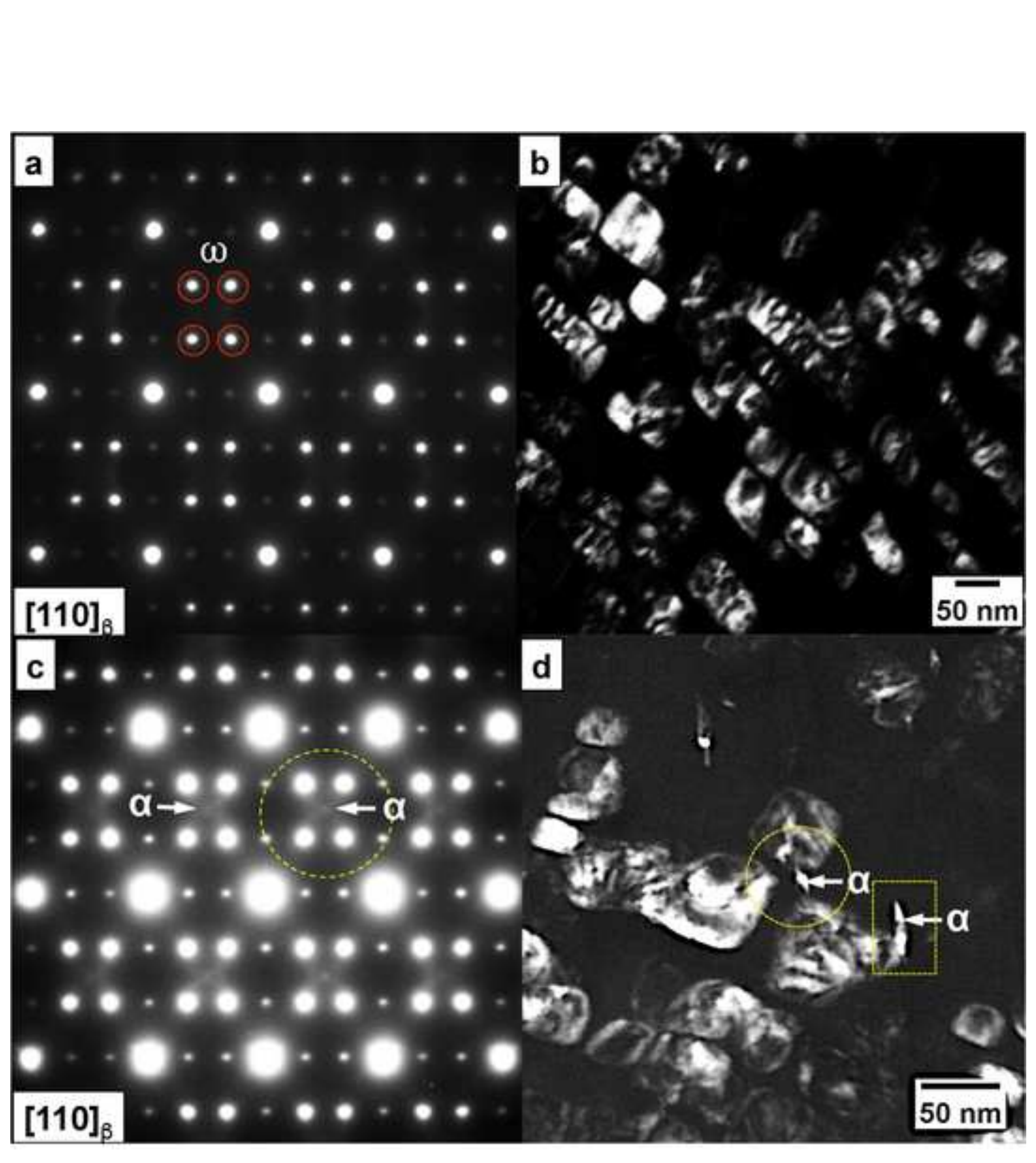

\section{Figure 1}
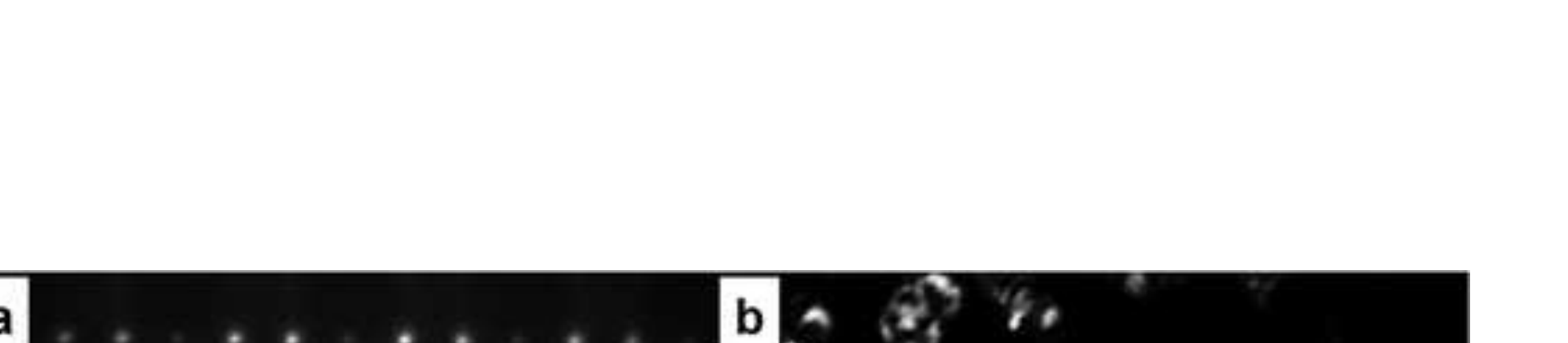





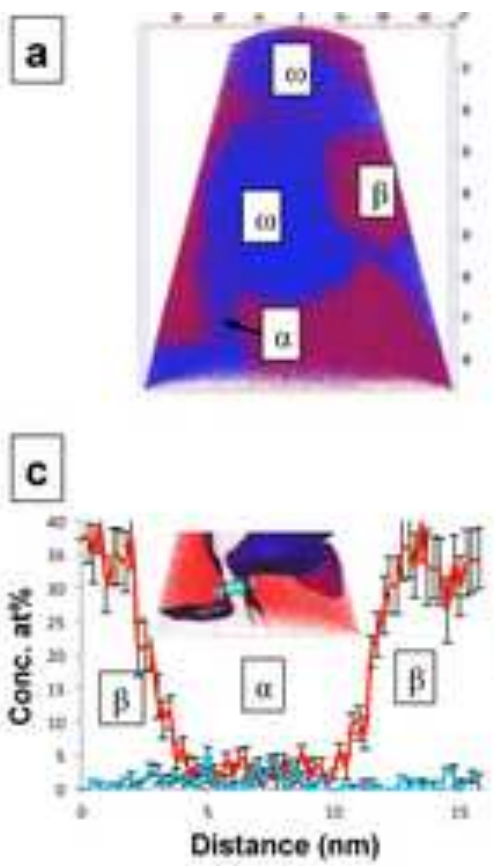

a
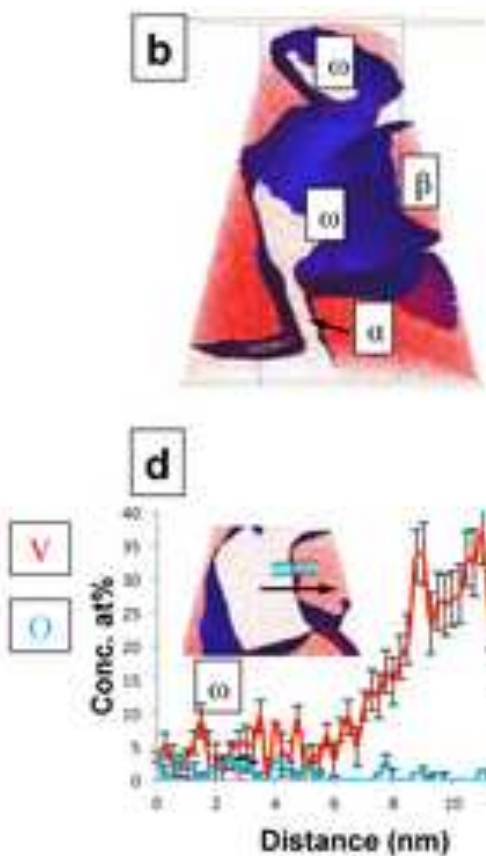
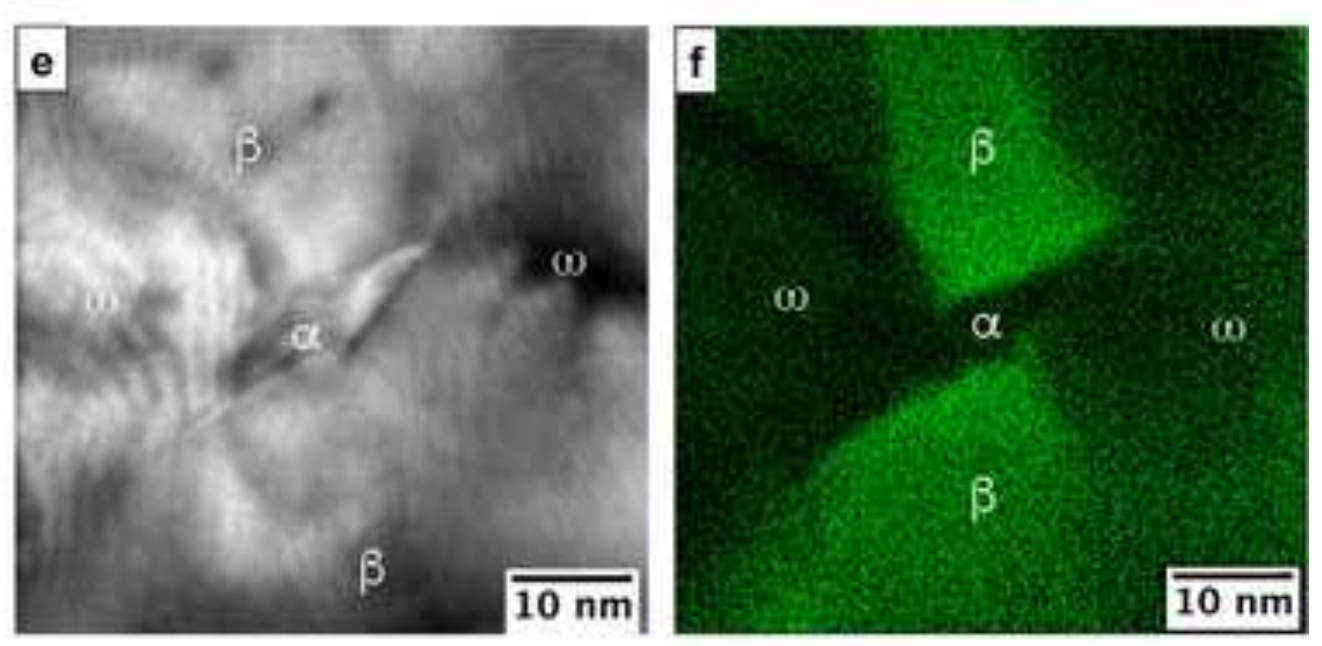

d

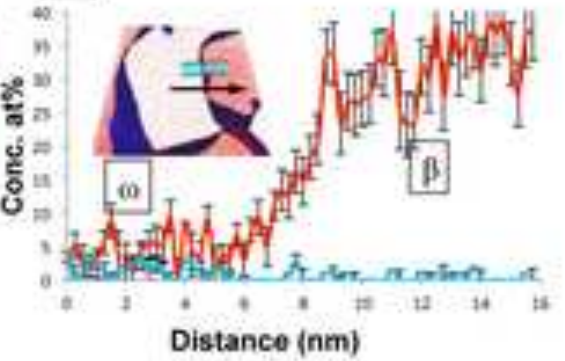

Distance (nen) 


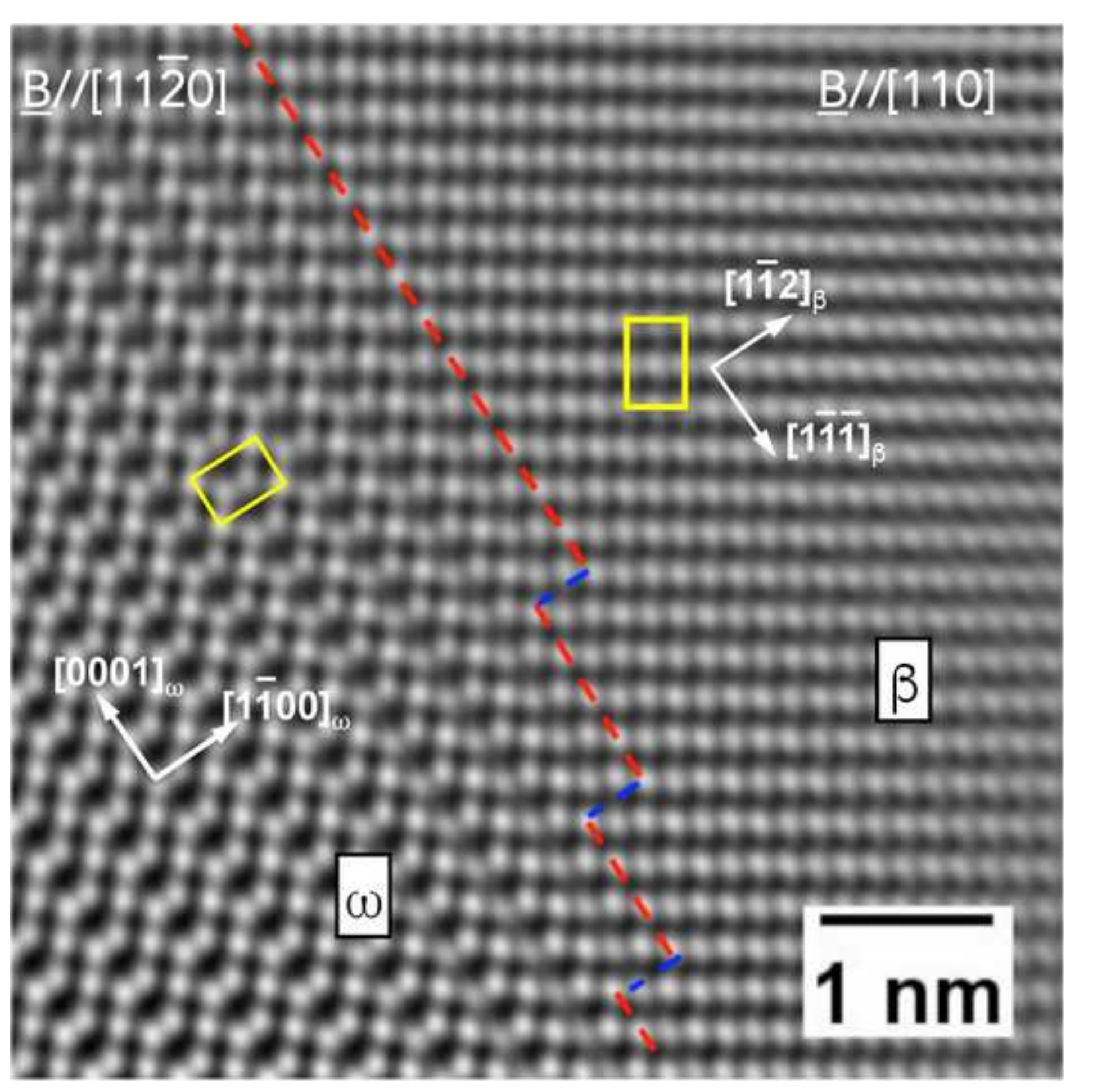

Figure

B/N1120]

112

$\mathrm{B} / /[110]$

3

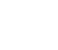

(

.

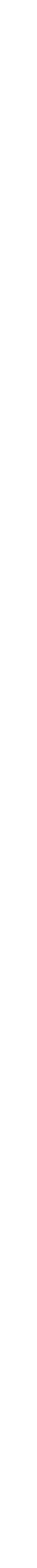



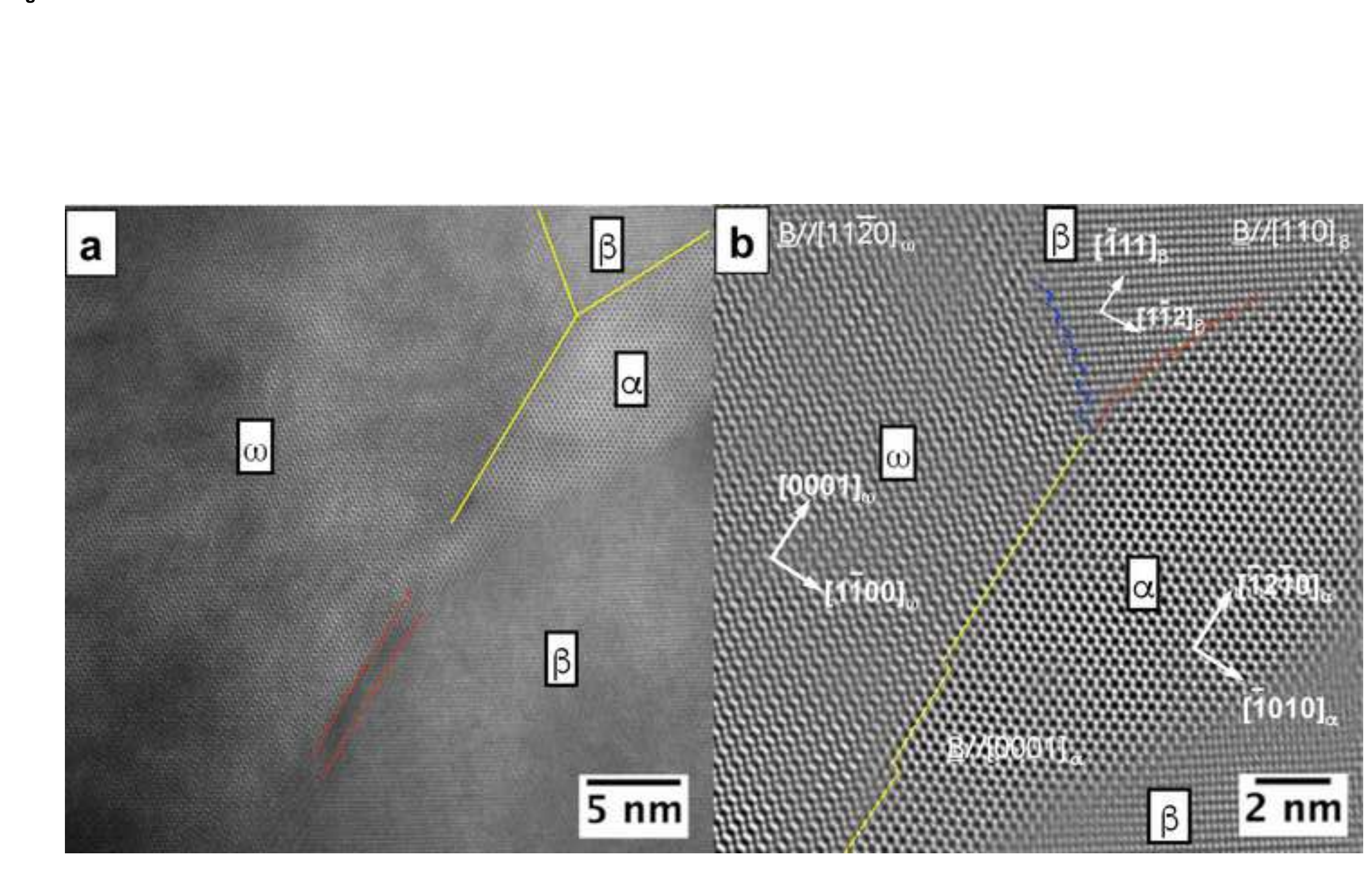

\section{.}

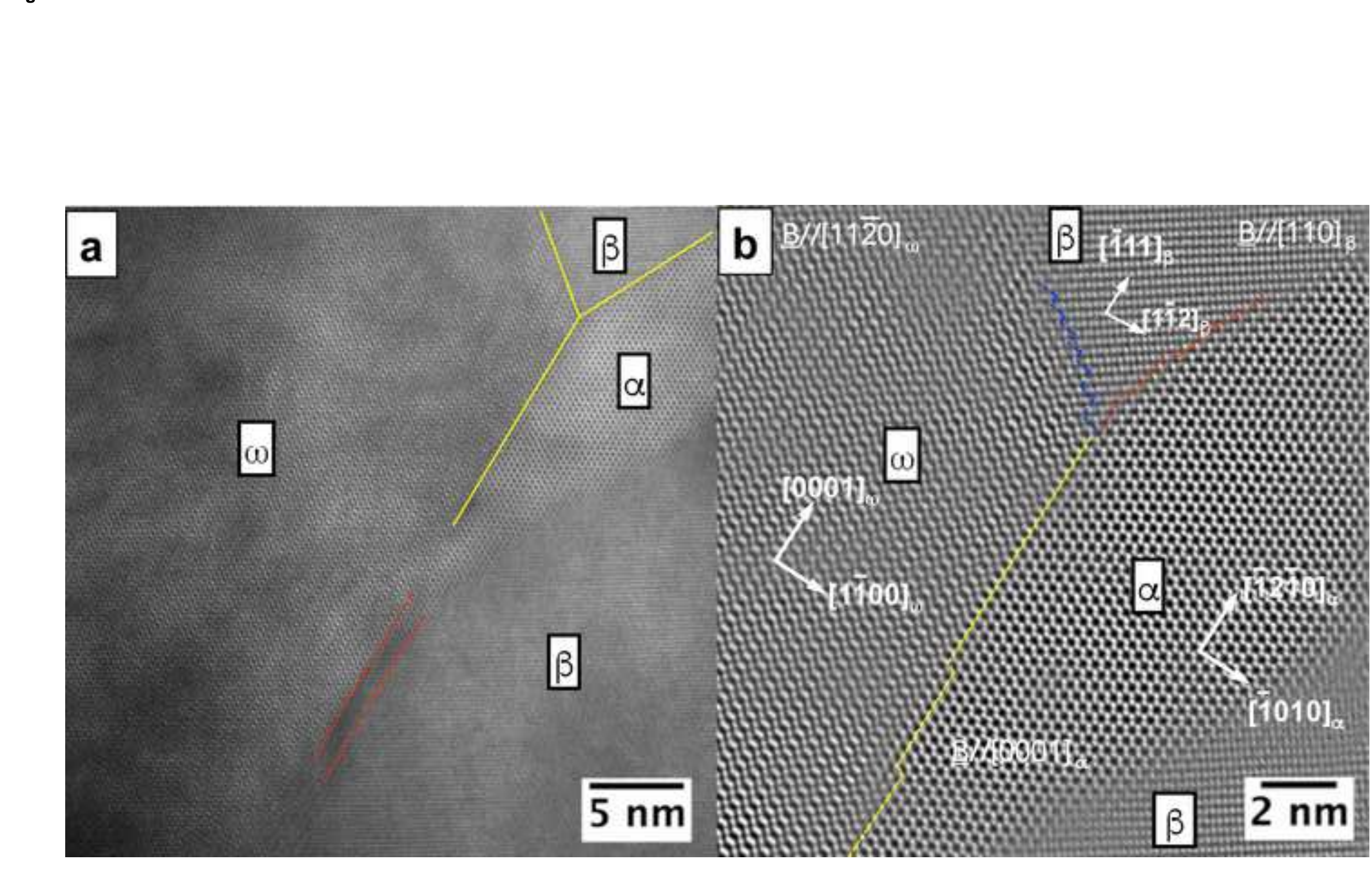

a

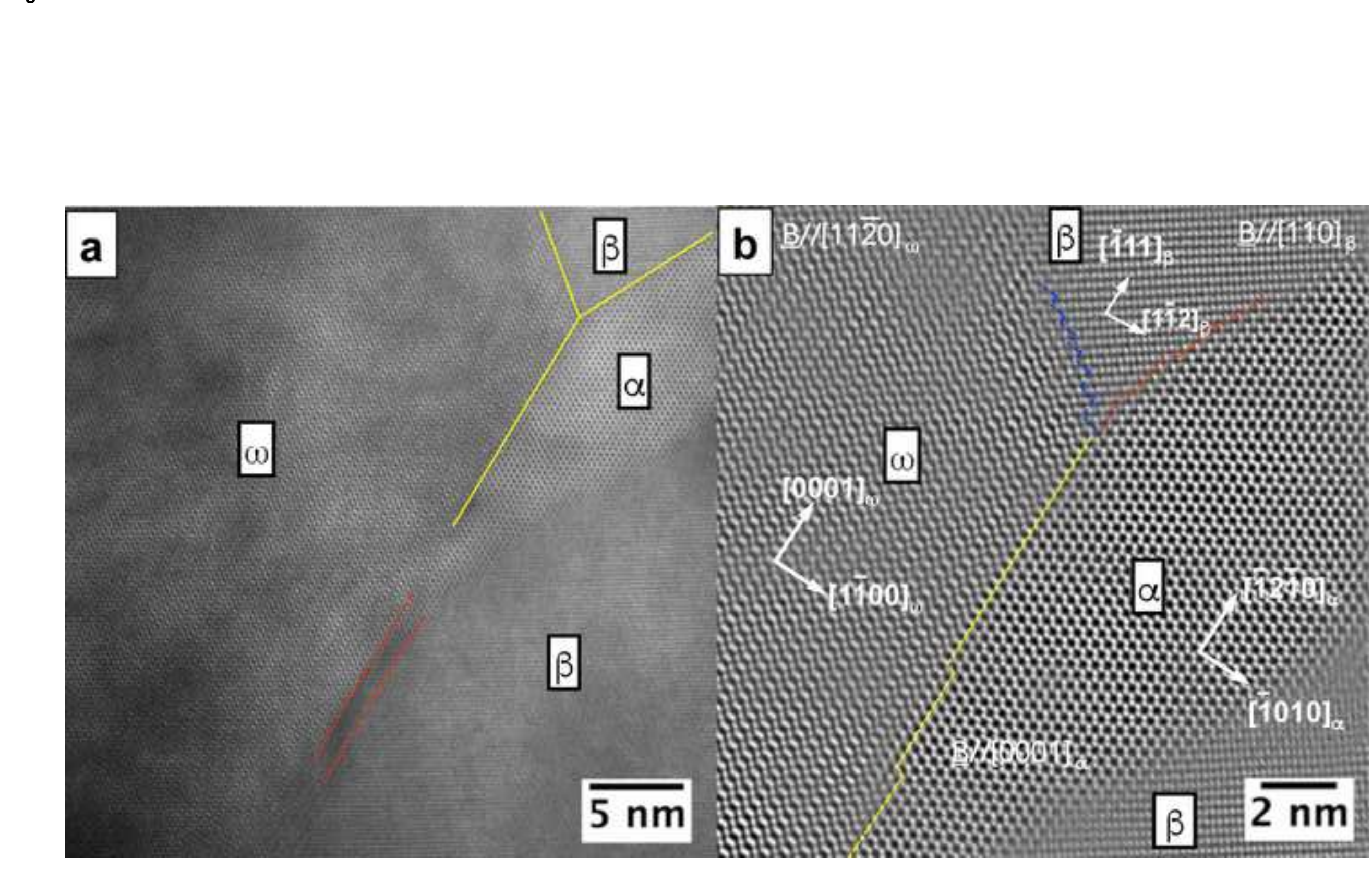



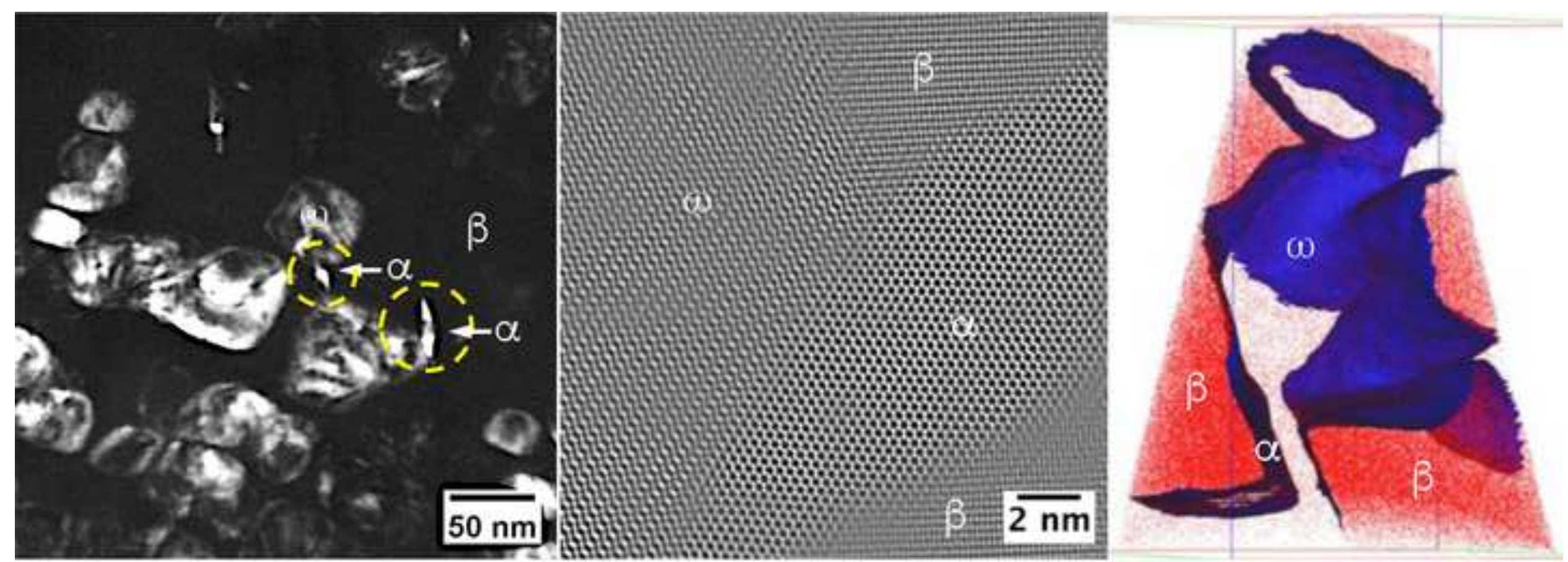